\section{Using Membrane-Supported Liquid-Liquid Extraction for the Measurement of Extraction Kinetics}

\section{Wolfgang Riedl ${ }^{*}$, Daniel Mollet, and Gerhard Grundler \\ ${ }^{*}$ Correspondence: Prof. Dr. W. Riedl, University of Applied Sciences Northwestern Switzerland, School of Life Sciences, Institute for Chemistry and Bioanalytics, Gründenstrasse 40, CH-4132 Muttenz, Tel. +4161467 4496, E-mail: wolfgang.riedı@fhnw.ch}

Abstract: Membrane-supported liquid-liquid extraction uses artificial membranes for the generation of a phase interface between the two liquid phases involved in extraction. Additional equipment for the generation of droplets as well as phase separation afterwards is no longer necessary. Since the membranes used for this special type of extraction are quite well described concerning thickness, porosity, tortuosity and material it is possible to generate information about the diffusion coefficient of the component to be extracted within the preferred solvent from extraction trails easily. This article describes an experimental set-up for both the proof of principle of membranesupported liquid-liquid extraction and, using a dedicated computer-aided data treatment, how to calculate the overall mass transfer coefficient as well as the diffusion coefficient for a given system within moderate testing duration.

\section{Keywords: Caprolactam - Diffusion coefficient · Membrane} extraction - Overall mass transfer coefficient

\section{Introduction}

Membrane-supported liquid-liquid extraction (memex) has been an interesting alternative to conventional liquid-liquid extraction (e.g. with agitated columns) for years. ${ }^{[1,2]}$ Especially if formation of emulsion takes place, memex has advantages over conventional technologies, since mixing of one liquid phase into the other is not necessary. Hence, emulsification does not occur and no phase separation is required.

Compared to conventional liquid-liquid extraction, the surface for mass transport is well described with memex. Hence, the overall mass transfer coefficient can easily be obtained by a simple test set-up. In parallel, for the investigated liquid-liquid system the diffusion coefficient of the extracted component can be obtained, too.

This makes memex interesting for both the proof of new technology for a given separation problem as well as an instrument for kinetic measurements.

\section{Basic Principles}

\section{Membrane-supported Liquid-Liquid Extraction}

Membrane-supported liquid-liquid extraction (memex) as an alternative extraction technology has been promoted for years. ${ }^{[3]}$ Some technical applications have already been reported. ${ }^{[1,3]}$ The principle of memex is shown in Fig. 1.

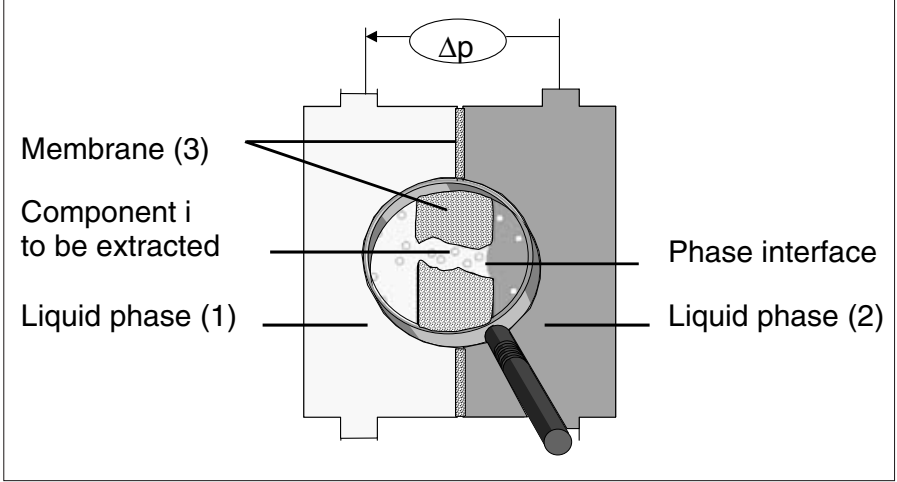

Fig. 1. Principle of membrane-supported liquid-liquid extraction (memex).

Like conventional liquid-liquid extraction, memex requires two liquid phases, feed (1) and solvent (2) phase, which are immiscible over a broad range of mixing ratio. The component(s) to be extracted must have a different solubility in both liquid phases, since the membrane itself shows no rejection for the main component(s). Having one of the two liquid phases on one side of a microporous membrane (3) and the other one on the back side of the membrane, phase contact can occur, if one of the two liquid phases enters into the membrane pores, driven by capillary force. Then, the phase interface is immobilized in the membrane pores where extraction takes place. For a stable process it is advantageous if one phase fully wets the membrane and the other does not. ${ }^{[1,4]}$ Since there is no distribution of one liquid phase into the other phase, formation of emulsions cannot occur and phase separation after extraction is not necessary. Thus, contrary to conventional liquid-liquid extraction, a density difference between the two liquid phases is not necessary.

A slight pressure difference (100-200 mbars) between the liquid phase not wetting the membrane and the phase filling the membrane pores is helpful to prevent bleeding-out of the wetting phase.

The transport mechanism of the component(s) to be extracted is diffusion, derived from a difference in solubility of the target component in both liquid phases. It can be described as an addition of the transport resistances for both the boundary layers $\left(\mathrm{k}_{1}\right.$, $\left.\mathrm{k}_{2}\right)$ and the membrane pores $\left(\mathrm{k}_{\mathrm{Mem}}\right)$. For the transport of a component $i$ from liquid phase 1 (wetting the membrane) to liquid phase 2 , with a given distribution rate VZ between phase 1 and phase 2, the overall mass transfer coefficient $\mathrm{K}_{\mathrm{i}}$ is given by Eqn. (1):[2,4]

$$
\frac{1}{\mathrm{~K}_{\mathrm{i}}}=\frac{1}{\mathrm{k}_{1}}+\frac{1}{\mathrm{k}_{\mathrm{Mem}}}+\frac{\mathrm{Vz}}{\mathrm{k}_{2}} \quad[\mathrm{~s} / \mathrm{m}]
$$

Since diffusion depends on temperature, path length and driving force, it is clear that high temperatures, thin membranes with low tortuosity degree and large differences in solubility of the main component(s) in both liquid phases will lead to a higher overall mass transfer coefficient. 


\section{Experimental Setup}

In order to test different liquid-liquid extraction systems concerning their applicability with membrane extraction, a lab-scale test unit was developed as shown in Figs 2 and 3.

The unit consist of two independent loops. Each loop has its own gear pump (0-2245 $\mathrm{ml} / \mathrm{min}$ flow rate) and a storage tank volume of $500 / 2000 \mathrm{ml}$. Both gear pumps feed into a membrane contactor. For this investigation, a $1.4 \mathrm{~m}^{2}$ liqui-cel ${ }^{\circledR} \mathrm{X} 40$ hollow-fibre membrane contactor was used. Absolute pressure in both loop circles can be adjusted and pressure drop over the membrane contactor can be measured at the entry and the exit of the device. Mass-transfer of both the main component and, to a certain extent, also the solvent, is measured by weight change in both storage tanks or, since pumped volume remains constant, via density measurement with a Coriolis flow meter in both liquid phases. Since required liquid volumes are reduced to a very minimum, tests can be carried out even with high priced substances (e.g. pharmaceutical applications).

Running both liquid phases loop wise (co-current or counter current), an equilibrium distribution of the component(s) to be extracted between the two liquid phases is reached after infinite time (see Fig. 4).

The decrease of concentration in the feed and increase of concentration in the extraction phase can be described quite well with a declining and a saturation curve. It is assumed that Eqn. (2)

$$
c_{i}(t)=c_{i, 0} \cdot e^{-\lambda \cdot t}+c_{i, \infty} \quad[\mathrm{mol} / \mathrm{l}]
$$

is valid for the description of the time-depended concentration of the extracted component in the feed phase and Eqn. (3)

$$
c_{i}(t)=c_{i, \infty} \cdot\left(1-e^{-\lambda \cdot t}\right) \quad[\mathrm{mol} / \mathrm{l}]
$$

is valid for the extract phase respectively (whereas $c_{i, 0}=$ concentration at beginning of the extraction, $\mathrm{c}_{\mathrm{i}, \infty}=$ concentration at infinite time).

Knowing the installed membrane area (A) as well as the distribution of the main target component(s) between the two aqueous phases (expressed by the distribution number VZ) and the actual concentration in both liquid phases given by Eqn. (2) and Eqn. (3), the overall mass transfer coefficient $\mathrm{K}_{\mathrm{i}}$ can be calculated using Eqn. (4). [4]

$$
K_{i}=\frac{d n}{d t \cdot A \cdot\left(c_{i, 1}-V Z \cdot c_{i, 2}\right)} \quad[\mathrm{m} / \mathrm{s}]
$$

The obtained overall mass transfer coefficient can then be used for a further scale-up of the liquid-liquid extraction process e.g. towards higher flow rate.

\section{Results and Discussion}

Several liquid-liquid extraction systems have been investigated in the past. Beside others, there are (solvent - component to be extracted - extract phase)

- toluene - caprolactam - water

- polyethylenglycol/water - lysozym - dextrane/water

- polyethylenglycol/water - bovine serum albumin (BSA) dextrane/water

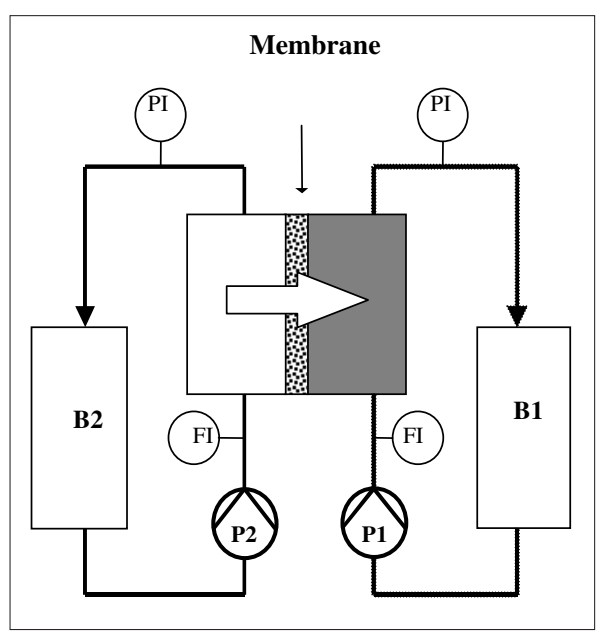

Fig. 2. Membrane extraction test-unit (principle).

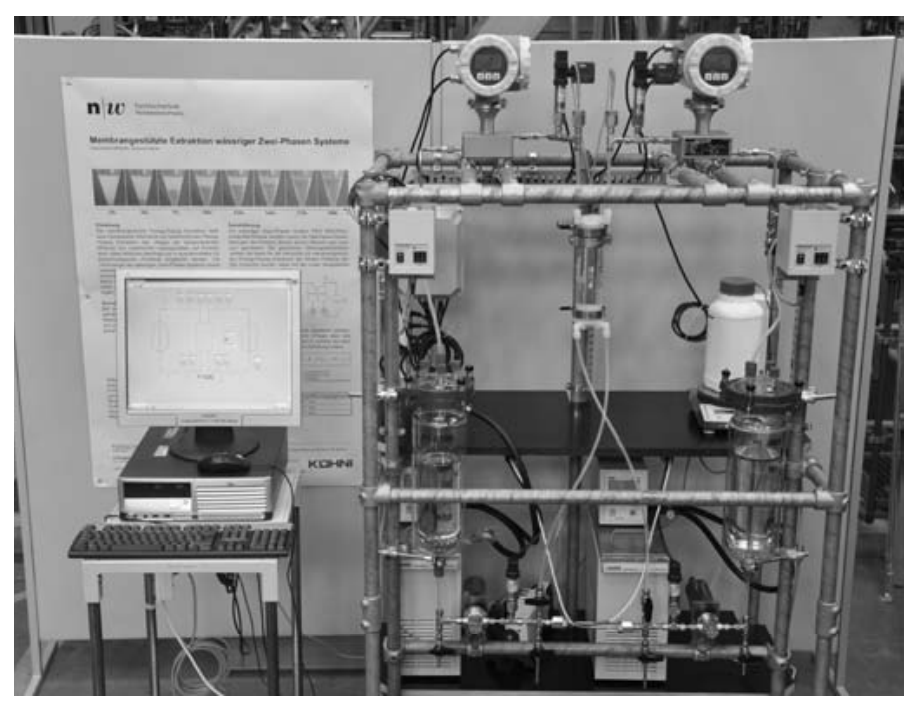

Fig. 3. Membrane extraction unit (incl. measurement and data acquisition).

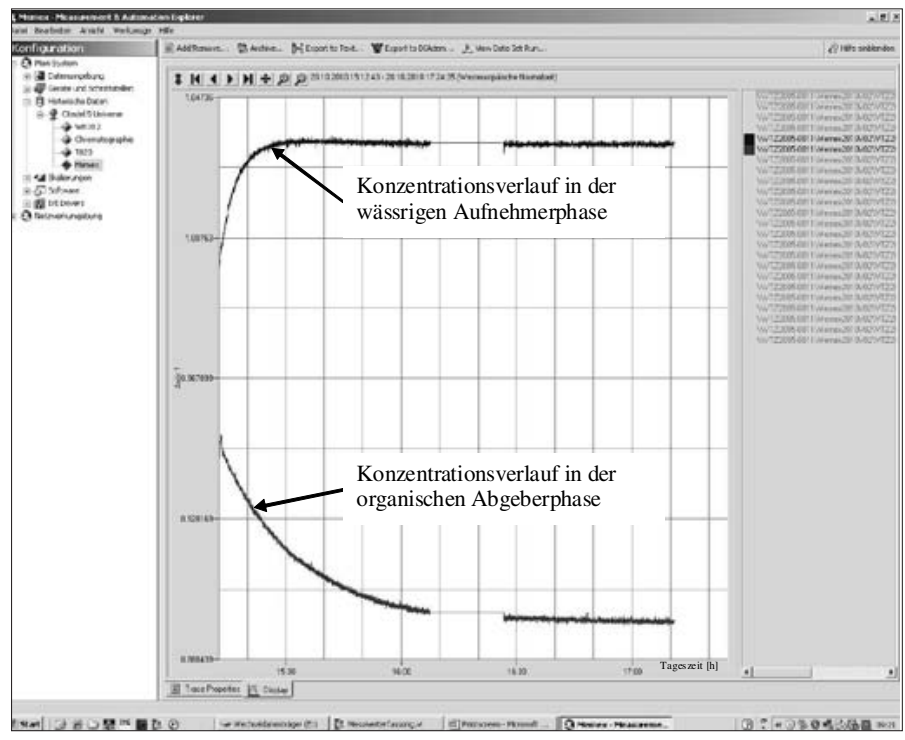

Fig. 4. Typical extraction curve.

- butylacetate - methylisobutylketone - water (under investigation)

Different types of membranes (hollow-fibres, capillaries) and materials were also investigated.

Each system could be extracted successfully, meaning no bleeding out of the phases occurred and only the components to be extracted were transferred into the other liquid phase until 
reaching the equilibrium concentration (given by the distribution number between the two liquid phases and the ratio between feed and solvent).

Within those experiments overall mass transfer coefficients were determined between $1.31 \cdot 10^{-5} \mathrm{~m} / \mathrm{s}$ and $1.78 \cdot 10^{-5} \mathrm{~m} / \mathrm{s}$ for caprolactam, $5.73 \cdot 10^{-6} \mathrm{~m} / \mathrm{s}$ and $3.47 \cdot 10^{-5} \mathrm{~m} / \mathrm{s}$ for lysozyme and $1.08 \cdot 10^{-5} \mathrm{~m} / \mathrm{s}$ for bovine serum albumin. For biomolecules such as urease, an overall mass transfer coefficient of $2.0 \cdot 10^{-6} \mathrm{~m} / \mathrm{s}$ is reported. ${ }^{[5]}$ For similar membranes as used for these trials, an overall mass transfer coefficient of $1.5 \ldots 2.8 \cdot 10^{-5} \mathrm{~m} / \mathrm{s}$ is reported for the extraction of citric acid between water and xylol.[6] Thus, the obtained overall mass transfer coefficients are in the same range as those described in literature and therefore can be used for a further scale-up within defined limits (e.g. maintaining hydrodynamic conditions).

For the system toluene-caprolactam-water, the dependency of the overall mass transfer coefficient on important parameters like temperature, velocity in both liquid phases, membrane type and thickness and transmembrane pressure were investigated. ${ }^{[4]}$ It could be shown that memex follows the rules of a diffusion driven process. Hence, especially temperature and length of diffusion path (e.g. tortuosity of the membrane, boundary layer thickness) do influence the overall mass transfer coefficient.

For all tested membranes, microscopic images (scanning electronic microscope) are available as well as information about average membrane thickness $\left(\mathrm{d}_{\mathrm{Mem}}\right)$, porosity $(\varepsilon)$ and tortuosity $(\tau)$. By applying defined fluid dynamic parameters (which are described elsewhere ${ }^{[4]}$ ), the transport resistance $\mathrm{k}_{\mathrm{i}, \mathrm{Mem}}$, derived from Eqn. (4), can be used for the calculation of the diffusion coefficient $\mathrm{D}_{\mathrm{i}}$ of the extracted component within the membrane wetting solvent, using Eqn. (5):[2]

$$
\mathrm{k}_{\mathrm{i}, \mathrm{Mem}}=\frac{\mathrm{D}_{\mathrm{i}}}{\mathrm{d}_{\mathrm{Mem}}} \cdot\left(\frac{\varepsilon}{\tau}\right)
$$

Following Eqn. (5) for the tested liqui-cel ${ }^{\circledR}$ membrane (porosity $\varepsilon=0,37$, tortuosity $=2,5, \mathrm{~d}_{\mathrm{Mem}}=25 \mu \mathrm{m},{ }^{[7]}$ ) and a overall mass transfer coefficient of $6.21 \cdot 10^{-6} \mathrm{~m} / \mathrm{s}$, a diffusion coefficient for caprolactam in toluene at $20^{\circ} \mathrm{C}$ of $1,0510^{-9} \mathrm{~m}^{2} / \mathrm{s}$ was calculated. A comparable diffusion coefficient of $1.13610^{-9} \mathrm{~m}^{2} / \mathrm{s}$ for caprolactam in trichloroethylene can be found in ref. [8]. Therefore this state-of-the art test set-up is applicable for the generation of data to calculate both the overall mass transfer coefficient and the diffusion coefficient of various components in different solvents at different temperatures.

\section{Conclusions}

Membrane-supported liquid-liquid extraction (memex) is an interesting alternative to conventional liquid-liquid extraction. The introduction of an artificial surface, the membrane, leads to an emulsion-free extraction, not requiring mixing and separating of the two liquid phases. Hence, equipment with rotating or moving parts is no longer necessary.

In addition, since the installed membrane area is well described, kinetic data of the component(s) to be extracted can easily be obtained with standardized experiments. It could be shown that the obtained overall mass transfer coefficients showed good agreement with those described in literature.

Since the experimental set-up requires little liquid volume, investigation of high-priced systems can also be carried out.

With the knowledge of the interdependence of diffusion processes from parameters such as temperature, flow conditions and membrane thickness, a scale-up not only to larger liquid volume but also to different parameters can be done.

Since membrane contactors are commercially available and even FDA-approved there are basically no real limitations for a technical realisation.

Received: March 25, 2011

[1] N. Li, A. Fane, W. S. Ho. T. Matsuura, 'Advanced membrane technology and applications', John Wiley \& Sons, New Jersey, 2008.

[2] T. Melin, R. Rautenbach, 'Membranverfahren', 2. Auflage, Springer Verlag, Berlin, 2003.

[3] J. Schneider, et al., 'Microporous Membrane Contactors in Industrial Separation Applications', Preprints des 8. Aachener Membran Kolloqiums, Forschungs-Gesellschaft für Verfahrens-Techik e.V., Mainz, 2001.

[4] W. Riedl, 'Membrangestützte Flüssig-Flüssig-Extraktion bei der Caprolactamherstellung', Shaker Verlag, Aachen, 2003.

[5] H. Walter, G. Johansson, 'Methods in Enzymology - Aqueous Two-Phase Systems', Academic Press, San Diego, 1994.

[6] R. Prasad, A. Kiani, R. R. Bhave, K. K. Sirkar, J. Membrane Sci. 1986, 26, 79

[7] Company information: membrane data sheet; http://www.liqui-cel.de/ product-information/data-sheets.cfm

[8] V. Hancil, V. Rod, M. Rosenbaum, Science 1970, 170, 1302. 